\title{
El tratamiento de la desigualdad económica en cibermedios internacionales: análisis de contenido desde la perspectiva del nuevo sistema híbrido
}

\author{
Rosa Pérez Arozamena \\ Universidad Europea del Atlántico \\ rosa.perez@uneatlantico.es \\ https://orcid.org/0000-0002-6125-1255
}

\author{
Javier Odriozola Chéné \\ Universidad Europea del Atlántico \\ javier.odriozola@uneatlantico.es \\ https://orcid.org/0000-0002-8595-1950
}

\section{Income inequality treatment in international cyber-media: content analysis from media hybrid system perspective}

\section{RESUMEN ABSTRACT}

La configuración de la opinión pública es un proceso en el que los medios de comunicación han tenido un papel fundamental. Sin embargo, desde la llegada de Internet, el rol de los medios de comunicación no nativos digitales (legacy

media) se ha visto cuestionado debido a la aparición de nuevos actores que han generado el establecimiento de un sistema híbrido de medios, compartiendo este nuevo espacio mediático con los medios tradicionales. En este contexto, el objetivo de este artículo es construir una herramienta metodológica basada en el análisis de contenido que profundice en los cambios que se están desarrollando en los cibermedios para contribuir a explicar cómo el proceso de producción periodística se está transformando. En una segunda fase los resultados obtenidos se complementarán con entrevistas en profundidad para obtener una visión completa de los procesos, paliando las plausibles deficiencias de la técnica selccionada, centrada en el producto final. La propuesta se centra en el estudio de la desigualdad económica, con el fin de profundizar en la construcción, evolución e importancia del concepto en la agenda cibermediática a través de una herramienta de carácter longitudinal (2009-2018) y aplicable en un contexto internacional.

PALABRAS CLAVE

Cibermedios; Desigualdad económica; Sistema híbrido de medios; Análisis de contenido; Producción periodística; Legacy media
The media have played a key role shaping the public opinion. However, since the burst of the Internet, the role of old media (legacy media) has been questioned due to the emergence of new contributors that have led to the establishment of a hybrid media system.

In this context, the objective of this communication is to build a methodological tool, based on content analysis. This tool goes in depth on changes that are being developed in the cyber-media to help to explain how the journalistic production process is being transformed. In this way, at a second stage, the results obtained will be complemented by in depth interviews to obtain a complete understanding of the processes. This process will avoid the potential deficiencies of the selected technique, focused on the final product. To this end, this design focuses on the study of income inequality, to delve into construction, evolution and importance of the concept in the cyber-agenda through a longitudinal tool (2009-2018) and applicable in an international context.

KEYWORDS

Cybermedia; Income Inequality; Hybrid Media System; Content Analysis; Journalistic Production; Legacy Media 


\section{Introducción}

En la actualidad, la construcción de la opinión pública se asienta en un ecosistema en el que las relaciones entre sus integrantes -actores políticos, medios de comunicación y ciudadanos- se están modificando, en gran parte, debido a la influencia de la comunicación digital, que provoca la mutación de los roles atribuidos a cada uno de estos integrantes tradicionalmente.

Esta afirmación que pretende reflejar la tesis central de Chadwick (2013) hace mención a un nuevo ecosistema: el sistema híbrido de medios, en el que el que conviven, compiten e interactúan las lógicas de los viejos medios con las de los nuevos (Chadwick, 2013. p. 207). Por tanto, el proceso de hibridación del sistema de medios no se caracteriza por un sometimiento por parte de los nuevos medios a las lógicas de los medios tradicionales; ni tampoco, por una revolución en la que las lógicas de los nuevos medios suponen la desaparición de las lógicas precedentes. Las lógicas mutan y se adaptan a esta nueva etapa de coexistencia. Para ello, resulta fundamental observar el mundo no desde una perspectiva dicotómica "o esto o lo otro", sino desde una perspectiva de convivencia "esto, pero también lo otro" (Chadwick, 2013, p. 5). Así, de esta nueva concepción de un sistema híbrido de medios y de su perspectiva inherente, se deriva el proyecto de investigación News, Networks and Users in the Hybrid Media System (Newsnet). Transformation of News and Media Industry in the Post-Industrial Era (RTI2018-095775-B-C43) en el que se enmarca este artículo.

La hipótesis de la que parte el proyecto es que la industria mediática tradicional, legacy media, está experimentando cambios en el marco de un sistema híbrido, en el que estos medios se ven obligados a convivir y competir con nuevos agentes mediáticos, de tal manera que estos cambios están recomponiendo sus características y funciones.

En este punto es importante definir lo que se entiende por "legacy media". Estos, también llamados old media o traditional media, son los nacidos antes de la irrupción masiva de Internet como medio de comunicación (Diel, 2017). De esta forma se incluyen periódicos impresos y empresas de noticias de radiodifusión (Westlund, 2013); así como estudios de cine y música, agencias de publicidad y televisiones (Logan, 2010; Peterson, 2003; Becker y Wehner, 2001), todos ellos bajo las prácticas y los valores noticia tradicionales. Sin embargo, es necesario tener en cuenta que, en esta nuevo ecosistema digital, irrupen una serie de medios que, si, bien presentan, unas características propias en sus rutinas de producción, derivadas de su configuración de nativos digitales, entroncan y comparten los principios que rigen el desarrollo de la profesión periodística (García-Santamaría, Clemente-Fernández y López-Aboal, 2013).

De esta hipótesis inicial, centrada en la transformación de la industria mediática, se derivan los tres objetivos generales que buscan profundizar en los cambios que afectan a nivel estructural de los propios medios, en el nivel de sus productos periodísticos y en el de las rutinas profesionales vinculadas al ejercicio de la práctica periodística.

En concreto, el objetivo principal de este artículo se vincula a los objetivos dos y tres. Ambos están centrados en determinar cómo el concepto, la naturaleza y los procesos de producción de la profesión periodística están cambiando; valiéndose para ello de los cambios observados a la hora de tratar temas de largo recorrido en la agenda mediática. Por ello, el mencionado objetivo general es construir una herramienta metodológica que permita observar los cambios que se han producido en el tratamiento informativo que los medios tradicionales realizan de un tema específico en sus productos periodísticos en relación con las características del nuevo sistema híbrido de medios.

La elección del tema viene determinada por el propio interés del grupo de investigación. Se elige el tema de la desigualdad económica por ser el eje central dentro del grupo de trabajo Income Inequality and Communication Group (en el que figuran la mayor parte del equipo de investigación del proyecto) como parte de Digidoc: Research Group on Digital Documentation and Interactive Communication de la Universitat Pompeu Fabra.

De esta manera una vez definido el tema de la agenda mediática que se analiza, desigualdad económica, se pretende construir una herramienta metodológica que permita dar respuesta a la siguiente pregunta general de investigación: ¿qué cambios, vinculados a la aparición de un nuevo sistema híbrido de medios, se observan en el tratamiento periodístico de la desigualdad económica por parte de los legacy media?

\subsection{La evolución de la desigualdad económica: de concepto académico a problemática social}

En 1971, Simon Kuznets obtuvo el Premio del Banco de Suecia en Ciencias Económicas en memoria de Alfred Nobel en reconocimiento a su contribución empírica para la interpretación del crecimiento económico, permitiendo una nueva y más profunda visión de la estructura económica y social y de los procesos de desarrollo vinculados a estas (The Nobel Prize, n.d.b). En el discurso de entrega del premio, desde la organización de los Premios Nobel, se recalcó la relevancia de 
sus estudios macroeconómicos para entender los procesos que contribuyen al desarrollo de cambios sociales (The Nobel Prize, n.d.a). En una de sus investigaciones, dieciséis años antes de la concesión del premio, Kuznets ya había medido la influencia de la desigualdad económica -income inequality - en el diferente crecimiento económico de los países (Kuznets, 1955), vinculándolo y estableciéndolo como un concepto fundamental dentro de sus teorías macroeconómicas que le harían merecedor del Premio Nobel.

A partir de entonces, el término empezó a cobrar relevancia en el mundo académico. Tradicionalmente, a la hora de definirlo se ha diferenciado la desigualdad económica entre naciones o países de la que hace referencia a las variaciones en los estándares de calidad de vida entre los ciudadanos de una población determinada (McKay, 2002). En el primero de los casos, el que se centra en las diferencias entre naciones, se vincula de manera habitual a otros conceptos como la globalización, la riqueza de los países o la pobreza (Seligson y Passé-Smith, 2008; Wilkinson y Pickett, 2009). En el caso de la desigualdad medida entre ciudadanos de una misma comunidad, se relaciona con la disparidad que provoca que unos individuos puedan acceder a ciertas condiciones de vida, mientras otros no (Ray, 1998. p. 170), vinculándose, habitualmente, a temas de salud pública, educación, violencia, movilidad social, sostenibilidad y pobreza (Redden, 2011; Wilkinson y Pickett, 2009). De manera paralela, otros investigadores han trabajado en destacar las fuerzas que contribuyen a reducir esta desigualdad, diferenciando entre fuerzas negativas como guerras, conflictos civiles, epidemias... y fuerzas positivas como la política, la educación pública, el acceso a la sanidad o los cambios tecnológicos (Milanović, 2016).

Si bien, el concepto de desigualdad económica, como se ha demostrado, Lleva presente en el entorno académico desde hace mucho tiempo, dos serán los momentos fundamentales en los que incrementa su relevancia en el conjunto de la sociedad, y por tanto su visibilidad.

El primero de ellos, en el contexto estadounidense, se produce en la década de los años 80 cuando la General Social Survey incorpora una serie de enunciados centrados en los efectos de la desigualdad económica dentro del bloque relativo a las diferencias sociales. Mediante una escala de Likert, los encuestados debían determinar el grado de acuerdo en relación con tres afirmaciones: (a) las diferencias de ingresos en EE. UU son demasiado amplias; (b) la desigualdad existe porque beneficia a los ricos y poderosos; y (c) las diferencias de ingresos son necesarias para la prosperidad de los Estados Unidos (McCall, 2015, p. 186). Los resultados de la encuesta longitudinal (1987, 1996, 2000, 2008 y 2010) consta- taban cómo los ciudadanos estadounidenses fueron aumentando su apoyo a los dos primeros enunciados y su desacuerdo con el tercero; hasta 1996, observándose un cambio de tendencia entre 1996 y el año 2000, y un repunte, a partir de este año, de la perspectiva que sostiene que la desigualdad económica en el país es excesiva, siendo promovida por los ricos y poderosos e innecesaria para la prosperidad de la nación (McCall, 2015, p. 190).

El segundo de los momentos se deriva de la crisis económica global de 2008. Dos libros, fundamentalmente, posicionan la desigualdad económica en el centro del debate público, estableciéndola como un elemento fundamental para entender las causas de la crisis y trascendiendo las fronteras del mundo académico. Dichas obras son The Spirit Level (2009) de Richard G. Wilkinson y Kate Pickett (2009); y Le Capital au XXle siècle de Thomas Piketty (2013). Asimismo, capital importancia han tenido otras, de entre las que destacan, la del Premio Nobel de Economía, Joseph Stiglitz (2012), The Price of Inequality, o las de Branko Milanović $(2010,2016)$, The Haves and the Have-Nots y Global Inequality.

De este modo, en un nuevo entorno condicionado por las consecuencias de la crisis económica, movimientos sociales como el movimiento Occupy Wall Street, en EE. UU (2012); la Primavera Árabe, en más de una decena de países del mundo árabe (2010-2012); el Movimiento 15-M en España (2011), o el grupo de los Chalecos Amarillos, en Francia (2018), incorporan la desigualdad económica como uno de sus ejes de actuación, ya sea de manera directa o transversalmente.

En este punto, es necesario tener en cuenta la capacidad de los medios de comunicación de contribuir a la visibilización de problemáticas/causas sociales (Strodthoff, Hawkins y Schoenfeld, 1985). Este proceso de "maduración" de una problemática se desarrolla en tres fases:

- La fase de desambiguación, en la que los principios de una causa social emergente se definen.

- La fase de legitimación, en la que los diferentes medios de comunicación regulan que aspectos de una causa social son relevantes para ser transformados en productos periodísticos.

- La fase de rutinización, en la que esos aspectos fijados como relevantes de la causa social son tratados de manera habitual y desde un enfoque específico por parte del medio de comunicación (Strodthoff et al., 1985, p. 135).

Este proceso se asienta en un modelo en el que, en un primer momento, los actores referentes de una problemática social se dirigen a medios de comunicación 
específicos, con el objetivo de focalizar la atención de un público específico en la propia conceptualización del problema -desambiguación-. Posteriormente, gracia a la labor de esos medios específicos, se traslada la atención al conjunto del ecosistema mediático - legitimación -, aumentando, de manera paralela, el público que percibe el problema como importante $y$, por tanto, el desarrollo de una conciencia social que hace que el tema incremente, de manera habitual, su importancia en la agenda mediática -rutinización- (Strodthoff et al., 1985, p. 137).

\subsection{El papel de los legacy media en la relevancia otorgada a la desigualdad económica}

Las empresas mediáticas tradicionales ya no tienen el control sobre la realidad informativa que atesoraron durante todo el siglo XX. La mayor capacidad de los ciudadanos, gobiernos, empresas... de contribuir al desarrollo de la opinión pública en un ecosistema en continuo cambio, provoca que los legacy media deban adaptarse a este nuevo escenario (Anderson, Bell y Shirky, 2012, p. 86), definido por Chadwick como un sistema híbrido de medios, donde estos medios deben compartir el espacio con nuevos actores mediáticos (2013).

El sistema híbrido se construye por medio de las interacciones entre las lógicas de los viejos y nuevos medios, haciendo referencia a las tecnologías, estructuras de producción, normas, comportamientos y formas de organización que caracterizan a cada uno de estos actores (Chadwick, 2013, p. 4). La coexistencia de ambos modelos, con sus características específicas, provoca que tanto unos como otros se adapten, interactúen con el otro y, en definitiva, co-evolucionen (Chadwick, 2013, p. 59). Es decir, surge un nuevo escenario en el que, por un lado, la combinación de cambios en la naturaleza de la audiencia y en la aproximación de esta a los medios con las características estructurales de los medios tradicionales, por otro, generan el surgimiento de nuevas formas híbridas de mediación (Chadwick, 2013, p. 5).

En este contexto, donde se desarrollan nuevas formas híbridas de mediación, surgen nuevas oportunidades para actores sociales, relegados históricamente en los medios de comunicación tradicionales, de hacerse visibles y entrar a formar parte del proceso de producción periodística (Chadwick, 2013, p. 6) que desemboca en la configuración de la agenda de los medios (McCombs, 2006). Cabe recalcar, que desde la perspectiva de la agenda-setting, la importancia de los legacy se ha visto modificada, ya que ha sido potenciada por Internet, haciendo necesario conocer cómo se construye la agenda pública, de acuerdo las nuevas lógicas entre viejos y nuevos medios, constatando, por el momento, que "no hay una respuesta unívoca que indique si los nuevos medios les disputan la agenda a los medios tradicionales o si repiten su temario, menos aún si tienen la capacidad de establecer la agenda pública" (Aruguete, 2015, p. 161).

En dicho proceso de configuración de las agendas mediáticas, Downs (1972, p. 38) constató la dificultad de que un tema específico permanezca en el centro de las mismas durante un periodo prolongado, siendo habitual que aparezca como un tema relevante durante un periodo corto de tiempo para gradualmente desaparecer del centro de la atención pública. Específicamente, Downs estableció cinco etapas por las que, habitualmente, trascurren los temas en relación con su capacidad de generar la atención de la opinión pública:

- Fase del pre-problema, en la que el problema existe, pero no ha captado la atención de la opinión pública, solo la de un pequeño grupo de expertos o grupos de presión.

- Fase de descubrimiento y entusiasmo eufórico, en la que a consecuencia de una serie de acontecimientos dramáticos o de otras razones, la opinión pública fija su atención en el tema, considerando que la propia sociedad tiene los mecanismos para resolver el problema de manera rápida.

- Fase de asimilación de los costes asociados a la solución del problema. Se empieza a desarrollar una conciencia común de que la solución del problema irá ligada a una serie de costes económicos o sociales, por ejemplo.

- Fase de descenso gradual del interés público. Ante la confirmación de las dificultades para solventar el problema y los costes asociados, se produce un descenso en el interés del público en el tema.

- Fase del post-problema. En esta fase final, el tema se desplaza desde el centro de atención de la opinión pública a las fronteras, recuperando su importancia mediante acontecimientos episódicos (1972, pp. 39-40).

Este proceso en el que los temas acrecientan o disminuyen su relevancia en la agenda mediática se fundamenta en el principio de que la capacidad de la agenda tiene límites. De este modo, los temas se ven obligados a competir para captar la atención de los medios y, como consecuencia, de la propia opinión pública (Zhu, 1992, p. 829). Este límite viene fijado por los propios límites de la audiencia - tiempo, acceso a la información y capacidad psicológica-. Así bien, con la llegada de Internet el acceso a la información se facilita y el espacio mediático 
se vuelve infinito, los temas continúan compitiendo por su espacio debido a la capacidad de atención y al tiempo disponible de la audiencia (McCombs, 2006, p. 85).

\section{Marco teórico}

De esta manera, la investigación, al hilo de la pregunta general de investigación, busca dar respuesta a dos cuestiones que ayudan a determinar hasta qué punto sistema híbrido de medios condiciona los productos y procesos de producción de los legacy media sobre la desigualdad económica: el ciclo de atención del tema en la agenda de los medios y la contribución de estos a su visibilización.

RQ.1. ¿Cómo ha evolucionado la relevancia y tratamiento informativo de la desigualdad económica en los legacy media a lo largo del tiempo?

RQ.2. ¿Cuál es la contribución de los medios de comunicación a la visibilización de la desigualdad económica como una problemática social?

A estas dos preguntas específicas se añade una tercera que permite enfatizar en las posibles diferencias entre medios de comunicación de diferentes países o de diferente naturaleza: medios generalistas frente a medios especializados.

RQ.3. ¿Se observan diferencias en el papel de los legacy media a la hora de visibilizar y dar cobertura a la desigualdad económica en función de criterios geográficos o de la naturaleza del medio?

\subsection{La relevancia y tratamiento informativo de la desigualdad económica}

Para poder operacionalizar la relevancia del tema en la agenda de los medios se recurre al propio concepto de relevancia mediática. Su conceptualización no es unívoca en los estudios de la agenda setting, recurriendo en ocasiones, a indicadores externos, si un tema destaca sobre otros de la agenda, o a sus características internas (Aruguete, 2015, pp. 46-47). Así, a la hora de medir la relevancia de un tema en la agenda de los medios, se ha estudiado como un concepto unidimensional, por ejemplo, cuando se construye desde la perspectiva del número de noticias publicadas (Dearing y Rogers, 1996, p. 18) o como constructos multidimensionales que abordan la atención que conceden los medios al tema, su prominencia en las noticias donde se trata o la valencia o tono desde el que se aborda el tema (Kiousis, 2004, pp. 74-76).

H.1. La relevancia de la desigualdad económica en la agenda de los medios, definida por el número de noticias publicadas, evoluciona de acuerdo con las fases del ciclo de atención de los temas, configurando un bucle a medida que se desarrollan nuevos ciclos de atención.

H.1.1. La desigualdad económica tiene una relevancia escasa en los medios de comunicación, por lo que los periodos en los que el tema se encuentra en la periferia de la agenda mediática son más extensos que los periodos en los que constituye como una cuestión importante.

Sin embargo, es necesario tener en cuenta que, a la hora de determinar la capacidad de un tema de generar el interés de los medios, son varios los elementos que pueden influir. Los temas tratados, los actores participantes, el encuadre desde el que se aborda o las lógicas de producción vinculadas a la cobertura de la desigualdad económica, se convierten en variables que permiten profundizar en la casuística que determina la evolución que el concepto tiene en la agenda de los medios.

En primer lugar, es necesario constatar con qué temas se vincula preferentemente la desigualdad económica. Tradicionalmente al tratar este concepto, se ha relacionado con temas de sanidad, educación, medio ambiente, pobreza, desempleo, seguridad, género, migración o nuevas tecnologías (Wilkinson y Pickett, 2009). Sin embargo, la capacidad de que un tema entre a formar parte de la agenda mediática en ocasiones, está vinculada a la relevancia que esa cuestión tenga en la agenda política. Si bien, la relación entre estas agendas y sus consecuencias en la agenda pública, no tiene un único modelo de comportamiento (Soroka, 2002, p. 281), la política se ha configurado tradicionalmente como uno de los ejes de la agenda de los medios. En el caso de la desigualdad económica, la presión social sobre las instituciones políticas se configura como una de las fuerzas positivas que pueden disminuirla junto a otras de carácter negativo como las guerras o los conflictos civiles (Milanović, 2016, p. 56). Por tanto, resulta necesario conocer la vinculación de la desigualdad económica con sus temas habituales, pero también a su desarrollo político, así como a otras fuerzas que condicionan su evolución. Además, hay que reseñar el sentido polisémico del concepto al ser utilizado desde una perspectiva macro, para medir las diferencias entre países, como desde una perspectiva micro, centrada en las diferen- 
cias entre miembros de una misma comunidad (McKay, 2002).

H.1.2. La relevancia mediática de la desigualdad económica aumenta cuando se vincula a cuestiones políticas y a protestas sociales.

H.1.3. La relevancia mediática de la desigualdad económica aumenta cuando se vincula a diferencias entre miembros de una misma comunidad o a diferencias entre comunidades que geográficamente se ubican en el área de influencia del medio de comunicación.

En segundo lugar, resulta fundamental conocer los actores sociales vinculados al concepto de desigualdad económica. La noticiabilidad de los acontecimientos ha estado marcada, tradicionalmente, por la relevancia de sus protagonistas (Galtung y Ruge, 1965; Golding yElliott, 1979; Harcup y O'Neill, 2017), siendo las instituciones políticas uno de los actores más legitimados por los propios medios de comunicación contando, por tanto, con una mayor visibilidad mediática (Thompson, 2005). Sin embargo, en el sistema híbrido de medios, nuevos actores, que tradicionalmente no tenían visibilidad, de acuerdo con las lógicas de los medios tradicionales, aumentan su capacidad de interactuar con los medios; de tal manera que los medios de comunicación se relacionan, no solo con los actores políticos habituales, sino que incorporan otros como activistas, blogueros o agencias de relaciones públicas (Chadwick, 2013, p. 87).

H.1.4. La escasa relevancia de la desigualdad económica en la agenda de los medios provoca una mayor diversidad de actores; si bien, los medios tienden a centrar la atención en aquellas noticias que se relacionan con actores políticos tradicionales.

Por último, se ha de tener en cuenta que la capacidad de los medios de comunicación no se limita a determinar qué temas deben centrar la atención del público, sino los significados que el público da a estos temas (McCombs y Evatt, 1995). De esta manera, los medios subrayan determinados aspectos, aumentando la relevancia en el tratamiento informativo que se da del tema debido al encuadre realizado (Entman, 1993, p. 53), fijando su esencia (Gamson y Modigliani, 1987, p. 143). A la hora de estudiar los encuadres utilizados por los medios de comunicación, se puede diferenciar entre los encuadres episódicos, presentando el tema mediante ejemplos, casos o acontecimientos específicos; y encuadres temáticos, en el que el tema se aborda desde una perspectiva más general y contextualizada (Gross, 2008, p. 171). Otra forma de abordarlos es por la presencia en las noticias de determinados marcos generales: el conflicto, el interés humano, las consecuencias económicas, le evaluación moral y la atribución de responsabilidades (Semetko y Valkenburg, 2000, pp. 95-96).

Como consecuencia del dominio de determinadas lógicas a la hora de encuadrar los temas en el ecosistema mediático actual, las características vinculadas al desarrollo del infoentretenimiento se han ido extendido a todos los soportes (Labio-Bernal, 2008; Gómez-Mompart, 2009) y a todos los temas de la agenda mediática (Otto, Glogger y Boukes, 2017). Esta expansión del infoentretenimiento desde las denominadas soft news (Tuchman, 1978) al conjunto de la agenda mediática, provoca a su vez, una serie de cambios en el estilo de redacción y otros aspectos formales habituales en las hard news (Alencar y Kruikemeier, 2018). De esta manera, autores como Berrocal, Redondo y Campos (2014), consideran necesario detectar una serie de estilemas distintivos a la hora de contrastar la presencia del infoentretenimiento en los productos periodísticos (p. 89).

H.1.5. La desigualdad económica aumenta su relevancia mediática cuando confluyen una serie de estilemas distintivos del infoentretenimiento: explicación aislada del acontecimiento, dominio del conflicto, personalización del tema en un personaje central y uso de imágenes impactantes.

\subsection{La contribución de los medios en la visibilización de la desigualdad económica}

A la hora de operacionalizar la contribución que realizan los medios de comunicación a la visibilización de la desigualdad económica como una problemática social, se parte de cómo conciben este proceso Strodthoff, Hawkins y Schoenfeld (1985).

Según estos autores, en un primer momento, resulta necesaria una desambiguación de la problemática para una clara definición de la misma. En ella, los esfuerzos persuasivos de los actores, que tratan de aumentar la visibilidad del problema, se centran en unos medios de comunicación específicos, los cuales por sus propios objetivos editoriales vinculados a la problemática se dirigen a un público también específico (Strodthoff et al., 1985, p. 139). En segundo lugar, la problemática se legitima y se traslada a la agenda de los medios de comunicación generalistas con el objetivo de persuadir de su relevancia a una audiencia mayor (Strodthoff et al., 1985, p. 139). Así, se entra en una tercera fase, en la que, a medida que la cobertura del tema se asienta en los medios especializados y generalistas, su relevancia se incrementa, de tal manera que su capacidad para acceder a la agenda no dependerá exclusivamente del desarrollo de acontecimientos específicos vinculados a 
la misma (Strodthoff et al., 1985, p. 146). Durante todo este proceso, el mensaje evoluciona de acuerdo con tres niveles de abstracción: general, centrada en definir la problemática y su importancia; doctrinal, en la que se sientan las bases de la misma, sus causas y consecuencias; y sustantiva, en la que se establecen las cuestiones prioritarias o sustantivas a la hora de abordarla (Strodthoff et al., 1985, pp. 140-141).

H2. La visibilidad de la desigualdad económica en los medios de comunicación varía, de tal manera que si bien, en todos ellos se ha producido un proceso de desambiguación, que permite conocer la problemática y su relevancia, la cobertura varía entre medios, que la han incorporado en mayor medida mediante un mensaje más complejo que acaba vinculándola a ciertos temas prioritarios, y medios, que presentan una menor cobertura de la desigualdad económica con un menor desarrollo de las causas, consecuencias y temas prioritarios vinculados.

A la hora de estudiar la participación de los medios en el aumento de la visibilidad de la desigualdad económica como una problemática social, es necesario tener en cuenta los cambios acaecidos como consecuencia del sistema híbrido de medios. De este modo, Internet ha supuesto la transformación de la esfera pública (Masip, Ruiz-Caballero y Suau, 2019; Bruns y Highfield, 2015) configurando y consolidando distintos espacios mediáticos, vinculados a las nuevas audiencias. Las audiencias se configuran no solamente como un elemento activo en la interpretación de los productos periodísticos, sino acusadamente activas en cuanto a la producción de contenidos y debate público en entornos online, naciendo así el prosumidor. Conceptos como este prosumer (Toffler, 1980) identifican a la nueva audiencia muy vinculada a los primeros formatos participativos de la Web 2.0 (O'Reilly, 2009) como son los blogs, foros o wikis. Así bien, la blogosfera se ha convertido en un concepto común en el imaginario colectivo.

Dentro del marco de esta esfera pública en red (Benkler, 2006) coexiste otra dinámica que caracteriza el panorama periodístico actual. Tal y como se recoge en el Informe Anual de la Profesión Periodística (APM, 2018) la especialización de la información es una realidad de la industria periodística. Por tanto, y conscientes de todo ello, los medios de comunicación han incorporado espacios mediáticos como los blogs, en los que no sólo se fomenta la participación de la audiencia, sino que posibilita la especialización. De otro lado, los suplementos o secciones de información especializada se han consolidado.

H2.1. La cobertura de la desigualdad económica inicia su proceso de desambiguación en espacios específicos de los medios de comunicación, entre los que destacan blogs, secciones especializadas y suplementos para posteriormente extenderse a las secciones habituales de los medios de comunicación.

Otra cuestión surge a raíz de lo expuesto es aquella que atañe a las fuentes de información. En la dinámica de la nombrada Web 2.0, los medios han perdido el monopolio del poder simbólico, es decir, la habilidad de ser los protagonistas en los acontecimientos que narran, dejando en manos de otros la influencia en las nuevas acciones y eventos sociales (Thompson, 1998) propiciando la aparición de nuevos actores.

Asimismo, y siguiendo a Strodthoff et al. (1985) en su ciclo de maduración de los temas, la información sobre los contenidos provendrá en primera instancia de fuentes académicas, fuentes con un alto grado de especialización, por ende. De este modo, las fuentes académicas, como content curator (Bhargava, 2009; Guallar y Leiva-Aguilera, 2013), dejarán paso durante el proceso a otras fuentes hasta llegar a las habituales en las rutinas periodísticas: gobiernos, partidos políticos o sindicatos, por ejemplo.

H2.2. El proceso de desambiguación de la desigualdad económica es iniciado, principalmente, desde fuentes académicas, siendo posteriormente legitimado por fuentes provenientes de colectivos sociales y organismos supranacionales, para finalmente, incorporarse al lenguaje de las fuentes institucionales tradicionales.

Por otro lado, y continuando con esta lógica, si los temas son introducidos en espacios mediáticos especializados a través de fuentes ad hoc, el género informativo cederá, necesariamente, espacio en favor de la opinión. Esto abunda también en el marco del infoentretenimiento, ya que dentro de sus dinámicas hay cada vez una mayor preponderancia de la opinión, siendo la editorialización de la información (Lozano, 2004) uno de sus estilemas.

H2.3. La cobertura de la desigualdad económica se desarrollada, de manera preferente, en textos de opinión, durante la fase de desambiguación, para, posteriormente, desarrollarse en mayor medida en textos informativos o mixtos.

De esta forma, y atendiendo al ciclo de maduración (Strodthoff et al., 1985) la última fase es la rutinización, cuando el tema se convierte en habitual en la agenda de los medios y en la esfera pública. Siendo así, y según la economía de la atención en la que solamente un redu- 
cido número de temas, personas o grupos pueden captar la atención del público durante un tiempo prolongado (Thrall et al., 2008), dentro de las rutinas periodísticas se incorpora la lógica del trending. Por tanto, el tema se presenta usando etiquetas específicas con el fin de fijar la atención.

H2.4. Los periodistas han incorporado de manera tardía el concepto de desigualdad económica a su lenguaje.

H2.5. Los medios de comunicación han integrado el concepto de desigualdad económica mediante el uso de etiquetas específicas.

\section{Propuesta de análisis}

\subsection{Características de la herramienta metodológica}

Para determinar cómo el concepto, la naturaleza y los procesos de producción de la profesión periodística están cambiando en un tema de largo recorrido en la agenda de los medios como la desigualdad económica, se opta por diseñar un análisis de contenido cuantitativo, al ser una técnica completamente asentada a la hora de estudiar los contenidos generados por los medios de comunicación (Krippendorff, 2004, p. 17). Este estudio de los contenidos mediáticos ha sido abordado desde diferentes enfoques teóricos entre los que destacan aquellos vinculados a la configuración de las agendas mediáticas, como es el caso de la teoría de la agenda setting o del framing, por ejemplo.

En cuanto a las características específicas del análisis de contenido, en primer lugar, con el objetivo de dar respuesta a las preguntas de investigación centradas en estudiar, por un lado, la evolución de la relevancia y tratamiento informativo dado a la desigualdad económica en los legacy media; y, por el otro, la contribución de estos a la visibilización de esta problemática social, se configura un análisis de contenido cuantitativo de carácter longitudinal. Para ello, se opta por fijar un periodo de tiempo comprendido entre los años 2009 y 2018. Se opta por el año 2009 como punto de partida por ser considerado el año de partida de la última crisis económica global.

En segundo lugar, debido al carácter comparativo inherente a las preguntas de investigación, resulta necesario la configuración de una herramienta que permita constatar las diferencias entre los contenidos de los cibermedios analizados de acuerdo a su propia naturaleza y contexto geográfico. De acuerdo a este criterio se opta por elegir tres cibermedios, dos de carácter generalista y uno especializado en economía, de cuatro países diferentes: EE. UU (The New York Times, The
Washington Post y The Wall Street Journal); España (El País, ABC y Expansión); Francia (Le Monde, Le Figaro y Les Échos); e Inglaterra (The Guardian, The Telegraph y The Financial Times).

Por último, para dar respuesta a las hipótesis de investigación es necesario constatar que será analizado tanto el contenido manifiesto que hace referencia a aquellos elementos que pueden contabilizarse de manera directa en el texto, como el contenido latente, que no puede ser medido de forma directa pero que puede ser representado por uno o más indicadores (Neuendorf, 2002, p. 23) y que, por tanto, requiere un juicio de valor por parte del codificador a la hora de fijar la información en una categoría determinada (Sjøvaag y Stavelin, 2012, p. 216).

\subsection{Unidades de análisis y tipo de muestreo}

Una vez identificados los cibermedios que serán analizados, se determina que, las unidades de análisis específicas serán todos aquellos productos periodísticos publicados en estos cibermedios, entre el 1 de enero de 2009 y el 31 de diciembre de 2018, que contenga el concepto "desigualdad económica"1 en el conjunto del producto.

En cuanto al tipo de muestreo, la asiduidad en el uso del concepto dentro de los productos periodísticos determinará si se trabaja con toda la población, en caso de que no haya un uso continuado, o un muestreo basado en la de construcción semanas artificiales (Hester y Dougall, 2007), en caso de que la presencia del concepto tenga una mayor continuidad y permita trabajar con muestras representativas del conjunto de la población.

\subsection{Diseño del análisis de contenido}

Para el análisis de contenido se crea una herramienta metodológica organizada en tres grupos de variables: el contexto, la atención mediática y la visibilidad. Dichos elementos se operacionalizan por medio de diferentes dimensiones, indicadores y categorías que permitirán contrastar las hipótesis de investigación planteadas.

En primer lugar, las variables contextuales tienen por objetivo ubicar claramente las unidades de análisis de cara al proceso de codificación y permitir la posterior comparación entre cibermedios según su naturaleza, generalistas o especializados, o según su país, EE. UU, España, Francia o Inglaterra (ver tabla 1).

En segundo lugar, de cara a determinar la relevancia de la desigualdad económica y profundizar en cómo que constituye su ciclo de atención, se analiza la atención mediática desde diferentes dimensiones. Por un lado, se estudia la relevancia, medida como concepto unidimen- 
sional, según el número de productos publicado, y los temas a los que se vincula.

Por otro lado, se profundiza en la atención mediática de la desigualdad económica según los encuadres específicos y generales, los actores vinculados a la desigualdad y los estilemas que contribuyen al desarrollo del infoentretenimiento (ver tabla 2).

En tercer y último lugar, se profundiza en la presentación de la problemática en los cibermedios con el objetivo de conocer su aportación a la visibilización social de la desigualdad económica. Para ello, se trabaja desde las siguientes dimensiones: aportación del medio, del producto y de las fuentes que introducen el concepto (ver tabla 3).

\subsection{Fiabilidad de la herramienta metodológica y proceso de recolección y análisis del material}

Derivado del análisis del contenido, tanto manifiesto como latente, desarrollado por la herramienta metodológica, resulta necesario garantizar la fiabilidad o grado de acuerdo entre codificadores. Para ello, un 10 \% del total de las unidades de análisis serán codificadas por ambos investigadores aplicando el método Holsti, para aquellas variables donde la diversidad de categorías dificulta el acuerdo por azar y el índice Kappa de Cohen que contempla el acuerdo por azar debido al carácter dicotómico, sí o no, de las categorías (Neuendorf, 2002, pp. 149-151).

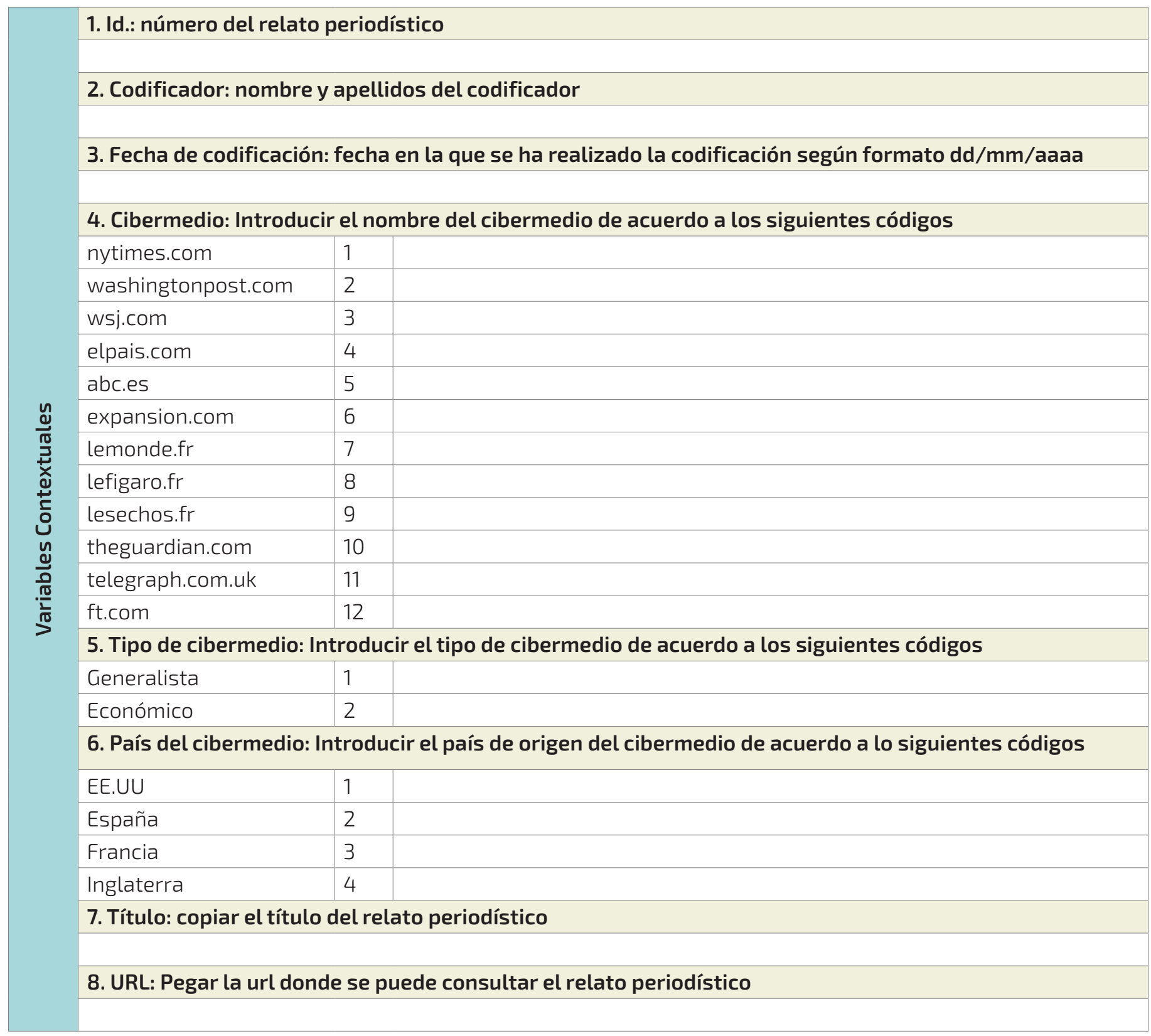

Tabla 1. Libro de códigos: variables contextuales. Elaboración propia. 


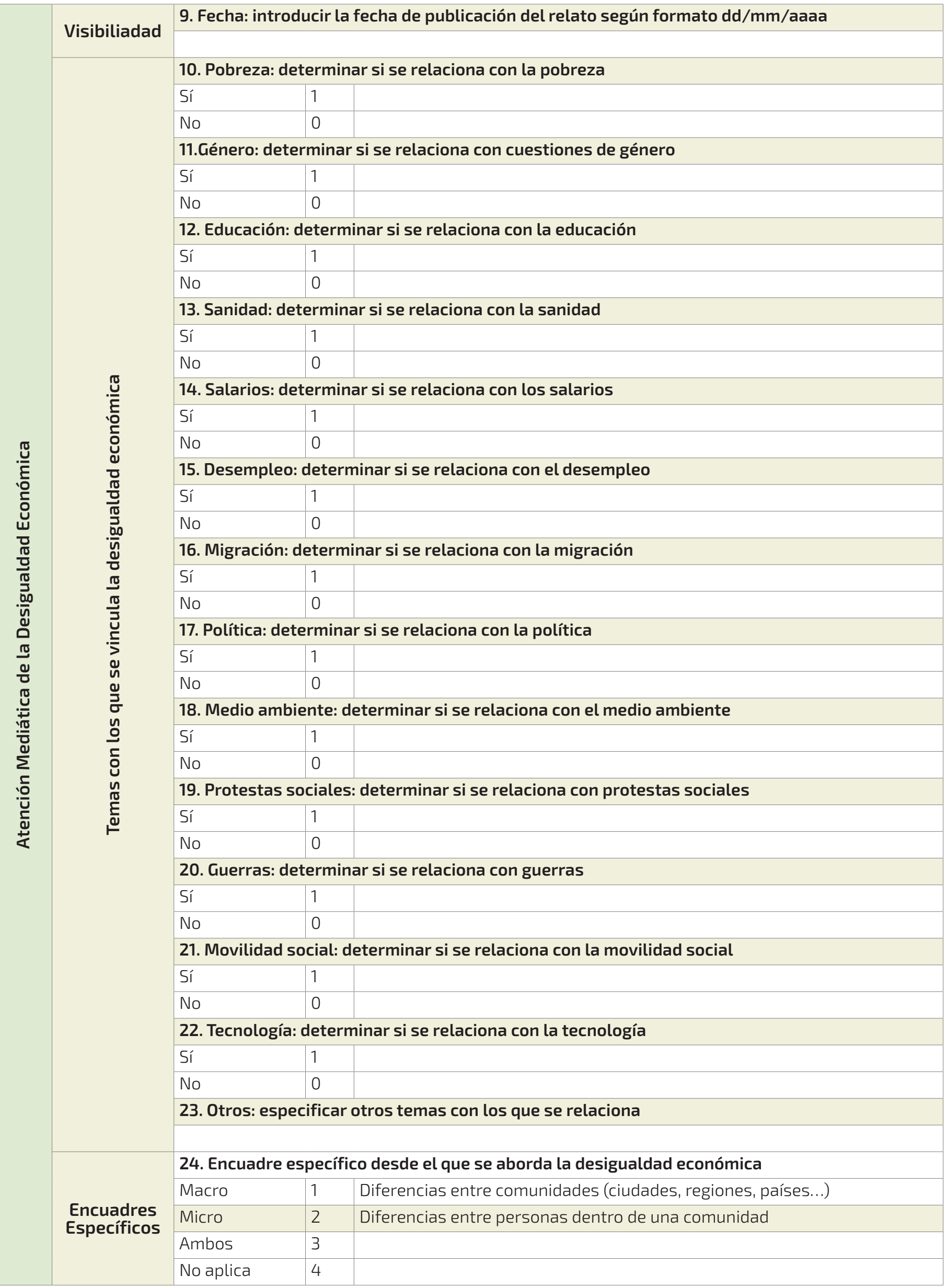




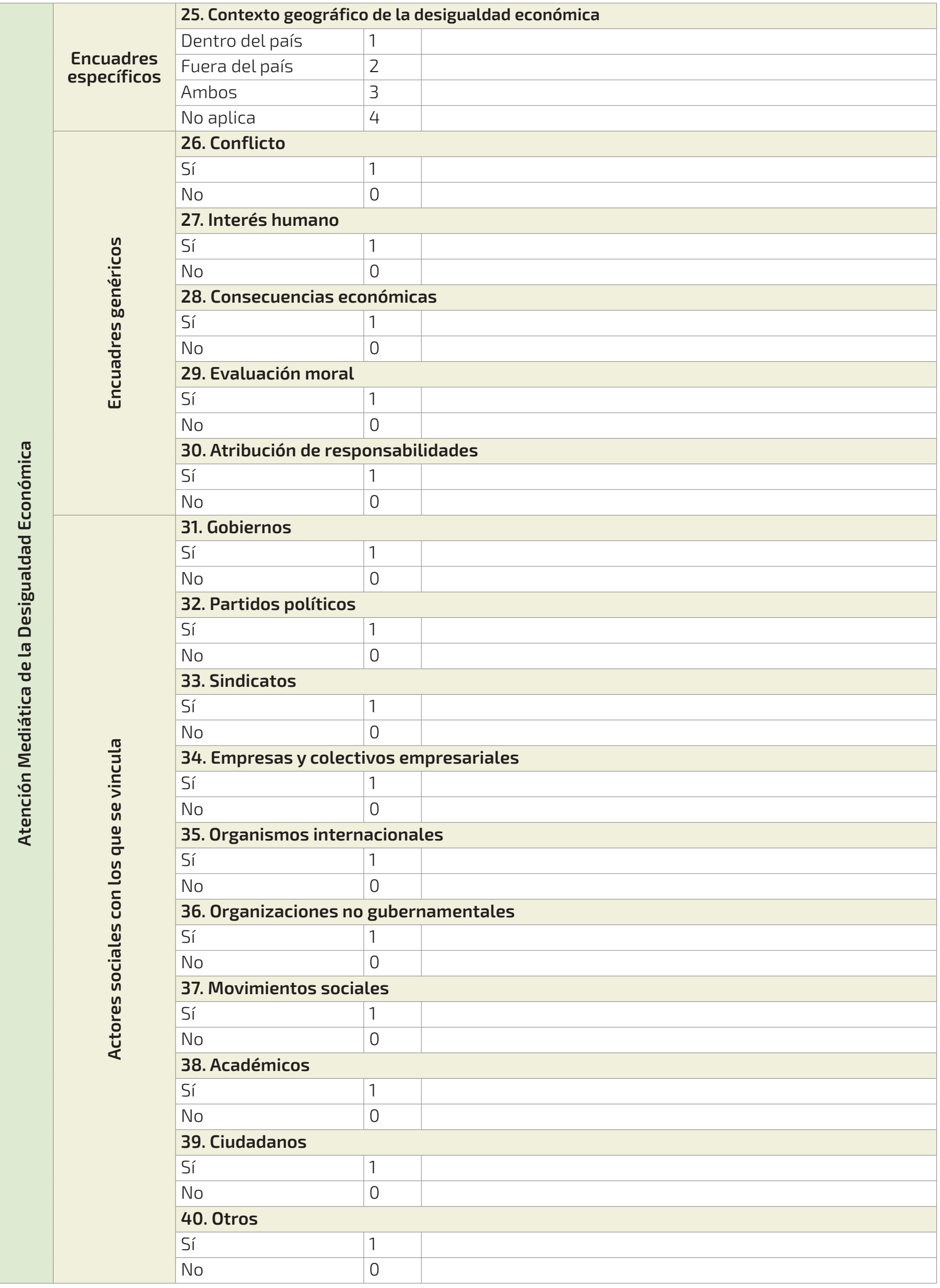




\begin{tabular}{|c|c|c|c|c|}
\hline \multirow{13}{*}{ 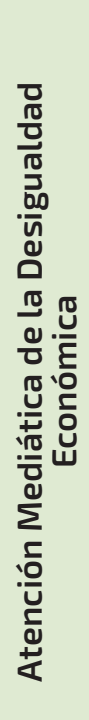 } & \multirow{13}{*}{ 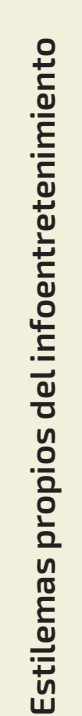 } & \multicolumn{3}{|c|}{ 41. Personificación: explicación aislada del suceso } \\
\hline & & Sí & 1 & \\
\hline & & No & 0 & \\
\hline & & \multicolumn{3}{|c|}{ 42. Dramatización: relevancia del conflicto por encima de otros encuadres } \\
\hline & & Sí & 1 & \\
\hline & & No & 0 & \\
\hline & & \multicolumn{3}{|c|}{ 43. Personificación: se presenta a un personaje como el elemento central } \\
\hline & & Sí & 1 & \\
\hline & & No & 0 & \\
\hline & & \multicolumn{3}{|c|}{$\begin{array}{l}\text { 44. Imagen impacto: piezas audiovisuales que generen impacto y fomenten la emotividad } \\
\text { y/o conflicto }\end{array}$} \\
\hline & & Sí & 1 & \\
\hline & & No & 0 & \\
\hline & & \multicolumn{3}{|c|}{ 45. Grado de infoentretenimiento acumulado: suma de los cuatro estilemas } \\
\hline
\end{tabular}

Tabla 2. Libro de códigos: variables atención mediática. Elaboración propia.

\begin{tabular}{|c|c|c|c|c|}
\hline \multirow{25}{*}{ 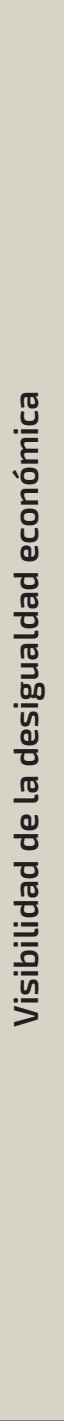 } & Visibiliadad & \multicolumn{3}{|c|}{ 9. Fecha: introducir la fecha de publicación del relato según formato dd/mm/aaaa } \\
\hline & \multirow{8}{*}{ 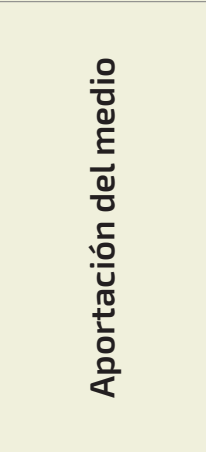 } & \multicolumn{3}{|c|}{ 46. Espacio mediático: introducir el lugar donde se ubica el producto periodístico } \\
\hline & & Secciones generales & 1 & \\
\hline & & Suplementos / especiales & 2 & \\
\hline & & Blogs & 3 & \\
\hline & & Productos de otras publicaciones del grupo mediático & 4 & \\
\hline & & \multicolumn{3}{|c|}{$\begin{array}{l}\text { 47. Uso de etiquetas: determinar si el producto se vincula a etiqueta específica de } \\
\text { "desigualdad económica" }\end{array}$} \\
\hline & & 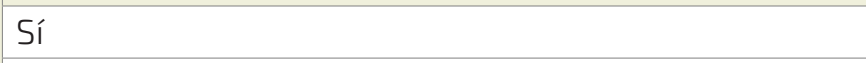 & 1 & \\
\hline & & \multirow{2}{*}{\multicolumn{3}{|c|}{$\begin{array}{l}\text { No } \\
\begin{array}{l}\text { 48. Uso del término por parte del periodista: en caso de que el texto esté firmado por un } \\
\text { periodista, señalar si este menciona el concepto }\end{array}\end{array}$}} \\
\hline & \multirow{7}{*}{ 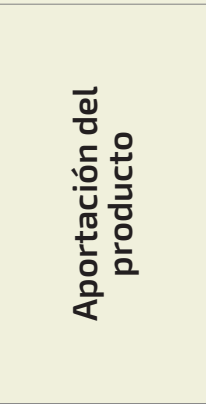 } & & & \\
\hline & & Sí & 1 & \\
\hline & & No & 0 & \\
\hline & & \multicolumn{3}{|c|}{ 49. Género periodístico: señalar que función domina en el producto } \\
\hline & & Informativa & 1 & \\
\hline & & Opinión & 2 & \\
\hline & & Mixta & 3 & \\
\hline & \multirow{9}{*}{ 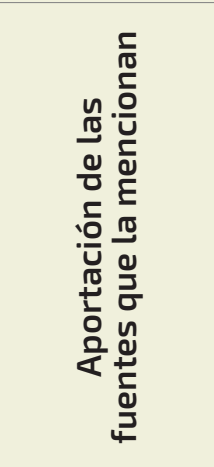 } & \multicolumn{3}{|l|}{ 50. Gobiernos } \\
\hline & & Sí & 1 & \\
\hline & & No & 0 & \\
\hline & & \multicolumn{3}{|l|}{ 51. Partidos políticos } \\
\hline & & Sí & 1 & \\
\hline & & No & 0 & \\
\hline & & \multicolumn{3}{|l|}{ 52. Sindicatos } \\
\hline & & Sí & 1 & \\
\hline & & No & 0 & \\
\hline
\end{tabular}




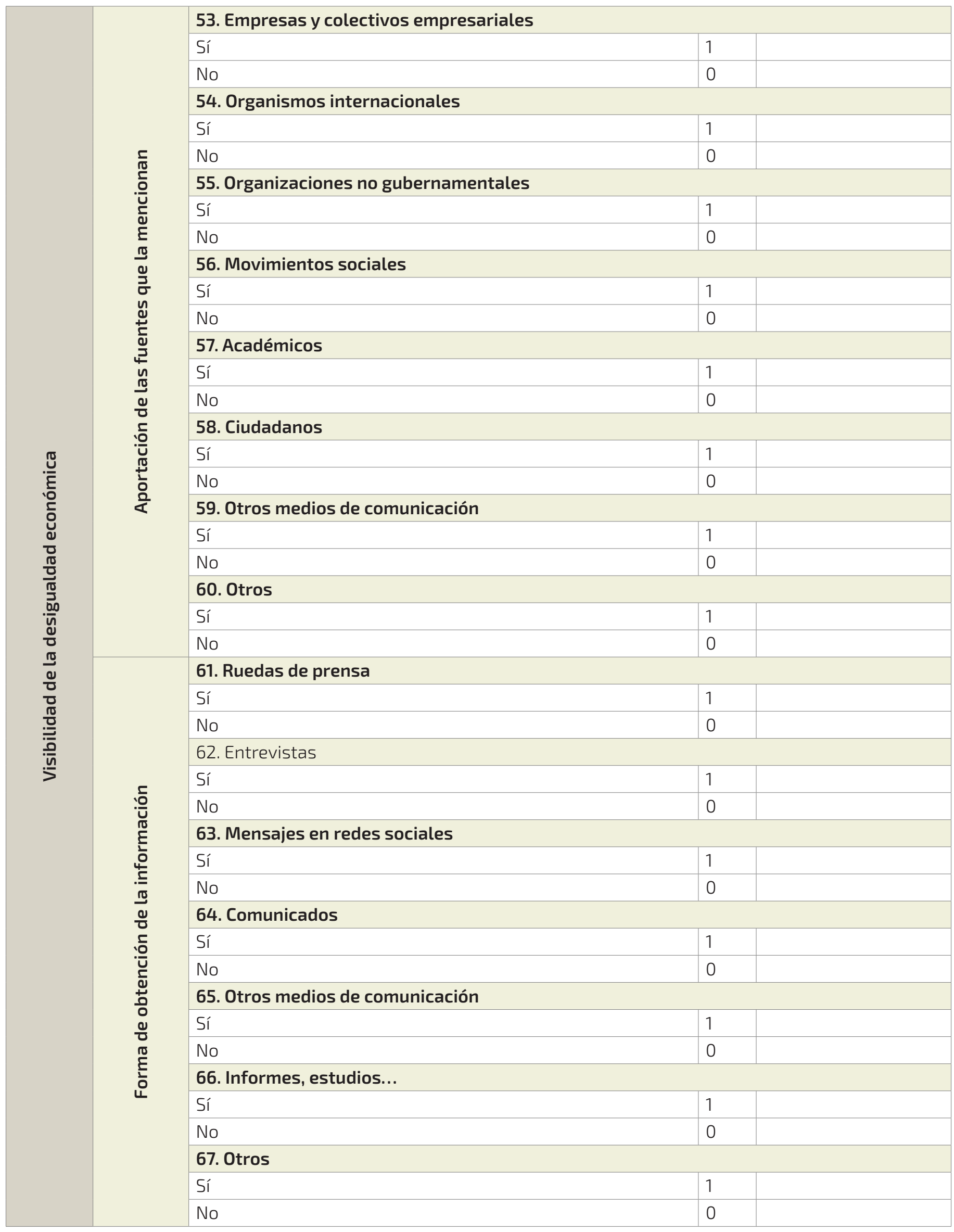

Tabla 3. Libro de códigos para la visibilidad. Elaboración propia. 
Por otro lado, la recolección de la información necesaria de las unidades de análisis se realizará mediante una hoja de cálculo donde se creará una base de datos y el libro de códigos que contribuya, junto a la propia formación de los codificadores, a asegurar un nivel óptimo de acuerdo. El análisis del material se realizará, posteriormente, mediante programas estadísticos que permitan resolver las hipótesis planteadas mediante el uso de la estadística descriptiva e inferencial.

\section{Resultados y discusión}

El diseño de la herramienta metodológica viene definido por un estudio preliminar (Odriozola Chéné, Pérez-Arozamena; Fernández-Planells, 2019; Pérez-Altable et al., 2020) desarrollado sobre el tratamiento de la desigualdad económica en siete cibermedios españoles a lo largo de 2018: 20minutos.com; elconfidencial.com; eldiario.es; elmundo.es; elpais.com; elperiodico.com; lavanguardia.com.

En dicho estudio se analizaron los 128 poductos periodísticos en los que se incluyó en su desarrollo el concepto "desigualad económica" con el objetivo de profundizar en la conceptualización, contextualización y tratamiento informativo del término.

En primer lugar, en relación con la conceptualización cabe destacar cómo el concepto de desigualdad económica, en los medios de comunicación analizados, se centra, principalmente, en señalar las diferencias entre personas de una misma comunidad; es decir, en su carácter micro, 71,1\%; frente a las diferencias que se dan entre comunidades, 28,9\%; enfocándose principalmente en contexto nacional, 47,5 \%, e internacional

Presencia Frecuencia (\%)

\begin{tabular}{|l|l|l|l|}
\hline \multicolumn{3}{|c|}{ Carácter de la desigualdad (n=128) } \\
\hline Micro & 37 & 28,9 \\
\hline Maro & 91 & 71,1 \\
\hline \multicolumn{3}{|c|}{ Tipo de desigualdad económica $(\mathbf{n}=\mathbf{1 2 8})$} \\
\hline Desigualdad de riqueza & 82 & 64,1 \\
\hline Desigualdad de renta & 60 & 46,9 \\
\hline $\begin{array}{l}\text { Desigualdad de con- } \\
\text { sumo }\end{array}$ & 19 & 14,8 \\
\hline \multicolumn{2}{|c|}{ Ámbito geográfico $(\mathbf{n}=\mathbf{1 1 8})^{2}$} \\
\hline Local & 1 & 0,8 \\
\hline Regional & 6 & 5,1 \\
\hline Estatal & 56 & 47,5 \\
\hline Internacional & 55 & 46,6 \\
\hline
\end{tabular}

Tabla 4. Conceptualización de la desigualdad económica en las informaciones analizadas en los los cibermedios. Fuente: Pérez-Altable et al. (2020).
46,6\%. Dentro de los tipos de desigualdad económica, será la desigualdad de riqueza, 64,1\%, la que prevalezca, seguida de la desigualdad de renta, 46,3\% (tabla 4).

En segundo lugar, desde una dimensión contextualizadora se pretende conocer quién y cómo se introduce el concepto, se define y, por tanto, se caracteriza. Cabe destacar que solo en un 26,1\% de los casos serán los periodistas, propios de medio o de la agencia que firma la información, quienes lo introducen. De esta manera, la desigualdad económica emerge, preferentemente, desde fuera del propio medio de comunicación por medio de otros colaboradores no periodistas autores de los textos, 28,4\%, o de las fuentes de información, 45,5 $\%$. Tanto cuando la mención procede de una fuente de la información como de un colaborador no periodista que ha redactado el texto, los expertos, 34,3\%, y los grupos de presión social, 16,2\%, prevalecen frente a otros actores con una mayor presencia en los procesos de

\begin{tabular}{|c|c|c|c|c|c|c|}
\hline \multirow[t]{2}{*}{ Procedencia } & \multicolumn{5}{|c|}{ 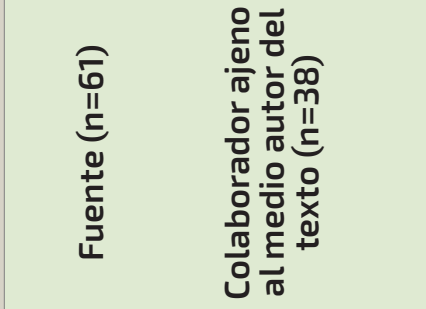 } & 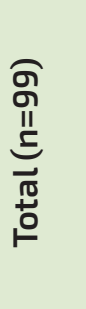 \\
\hline & $\begin{array}{l}\frac{\pi}{U} \\
\frac{c}{0} \\
\tilde{U} \\
\frac{1}{\square}\end{array}$ & $\begin{array}{l}\frac{0}{\pi} \\
\frac{0}{0} \\
\frac{0}{0} \\
\frac{0}{0}\end{array}$ & 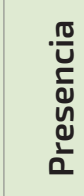 & 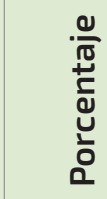 & 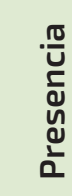 & 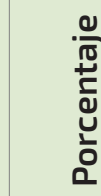 \\
\hline $\begin{array}{l}\text { Instituciones públi- } \\
\text { cas }\end{array}$ & 7 & 11,5 & 3 & 7,9 & 10 & 10,1 \\
\hline $\begin{array}{l}\text { Organismos inter- } \\
\text { nacionales }\end{array}$ & 8 & 13,1 & 1 & 2,6 & 9 & 9,1 \\
\hline $\begin{array}{l}\text { Partidos políticos y } \\
\text { sindicatos }\end{array}$ & 6 & 9,8 & 5 & 13,2 & 11 & 11,1 \\
\hline Empresas & 1 & 1,6 & 0 & 0,0 & 1 & 1,0 \\
\hline $\begin{array}{l}\text { Otras organi- } \\
\text { zaciones no } \\
\text { gubernamentales }\end{array}$ & 10 & 16,4 & 6 & 15,8 & 16 & 16,2 \\
\hline $\begin{array}{l}\text { Fuentes académi- } \\
\text { cas }\end{array}$ & 20 & 32,8 & 14 & 36,8 & 34 & 34,3 \\
\hline Periodista & 2 & 3,3 & 4 & 10,5 & 6 & 6,1 \\
\hline $\begin{array}{l}\text { Otros medios de } \\
\text { comunicación }\end{array}$ & 0 & 0,0 & 1 & 2,6 & 1 & 1,0 \\
\hline Ciudadanos & 0 & 0,0 & 1 & 2,6 & 1 & 1,0 \\
\hline Otros & 7 & 11,5 & 3 & 7,9 & 10 & 10,1 \\
\hline
\end{tabular}

Tabla 5. Procedencia de los autores de la mención cuando no es realizada por el propio periodista autor de la información ${ }^{3}$. Fuente: Pérez-Altable et al. (2020). 


\begin{tabular}{|l|l|l|}
\hline \multicolumn{1}{|c|}{$\begin{array}{c}\text { Tema principal de la } \\
\text { noticia (n=128) }\end{array}$} & Presencia & $\begin{array}{c}\text { Frecuencia } \\
\text { (\%) }\end{array}$ \\
\hline Salud & 2 & 1,6 \\
\hline Educación & 4 & 3,1 \\
\hline Medio ambiente & 5 & 3,9 \\
\hline Pobreza & 19 & 14,8 \\
\hline Desesmpleo & 3 & 2,3 \\
\hline Clases sociales & 17 & 13,3 \\
\hline Seguridad pública & 1 & 0,8 \\
\hline Salarios & 13 & 10,2 \\
\hline Igualdad de género & 17 & 13,3 \\
\hline Migración & 4 & 3,1 \\
\hline Impuestos & 4 & 3,1 \\
\hline Balance comercial & 8 & 6,3 \\
\hline Otros ${ }^{4}$ & 31 & 24,2 \\
\hline
\end{tabular}

Tabla 6. Tema principal de las informaciones analizadas en los cibermedios. Fuente: Pérez-Altable et al. (2020).

producción periodística en los medios tradicionales y en Internet (Philips, 2010, p. 99) (tabla 5).

De manera paralela, los temas a los que se vincula el concepto también se escapan de los habituales "temas-estrella" de las agendas mediáticas, destacando entre ellos: la pobreza, 14,8\%, la igualdad de género, 13,3 \% y la estratificación social, 13,3 \% (tabla 6).

En último lugar, su tratamiento informativo muestra que los atributos cognoscitivos centrales (Semetko y Valkenburg, 2000) desde los que se construye la desigualdad económica son variados y muy equilibrados, destacando: las consecuencias económicas, 28,1\%; el conflicto $25 \%$, el interés humano, 22,7 \% y la atribución de responsabilidad, 21,1\% (tabla 7).

Estos resultados permiten observar cómo el concepto no es habitual en el lenguaje de los propios periodistas, siendo introducido, preferentemente, por otros actores

\begin{tabular}{|l|l|l|}
\hline $\begin{array}{l}\text { Atributo cognoscitivo } \\
\text { central (n=128) }\end{array}$ & Presencia & Frecuencia (\%) \\
\hline $\begin{array}{l}\text { Atribución de } \\
\text { responsabilidad }\end{array}$ & 27 & 21,1 \\
\hline Interés humano & 29 & 22,7 \\
\hline Conflicto & 32 & 25,0 \\
\hline Moralidad & 4 & 3,1 \\
\hline $\begin{array}{l}\text { Consecuencias } \\
\text { económicas }\end{array}$ & 36 & 28,1 \\
\hline
\end{tabular}

Tabla 7. Atributos cognoscitivos centrales en las informaciones analizadas en los cibermedios. Fuente: Pérez-Altable et al. (2020). que actúan como fuente de información o como autores de un texto argumentativo o de opinión. Además, a la hora de definirse la desigualdad económica, se realiza, fundamentalmente, en relación con las diferencias de riqueza y renta entre miembros de una misma comunidad, vinculándose a temas relacionados con la pobreza, la igualdad de género y las clases sociales; si bien ya tiene cabida en cuestiones políticas y en otras áreas temáticas, no vinculadas tradicionalmente a esta problemática: arte deporte, cultura, ciencia y tecnología... Por último, no se trivializa el contexto del problema, primando el conflicto y las consecuencias económicas debido a la magnitud y el conflicto inherente al término.

Si bien estos resultados permitieron, en su momento, conocer la situación en dicho momento del tratamiento del concepto de la desigualdad económica en el panorama cibermediático estatal; no permitía ver cómo se fue construyendo el concepto a lo largo de la última década, una vez que cobró relevancia tras la crisis económica de 2008-2009 y si la reconstrucción del término en los cibermedios españoles es similar a la que se está produciendo en otros países.

Por todo ello, se plantea esta nueva herramienta metodológica de carácter longitudinal e internacional que modifica ciertos aspectos de la precedente para entender el tratamiento de la desigualdad económica desde teorías como el ciclo de atención de los temas en la agenda mediática (Downs, 1972) o los procesos de visibilización de problemáticas sociales (Strodthoff et al., 1985) y la adaptación de estos procesos al nuevo sistema híbrido de medios.

\section{Conclusiones}

Cabe reseñar que el principal aporte del presente artículo es la construcción de la propia herramienta metodológica. Herramienta, que tal y como se expone en el objetivo principal, permitirá observar la evolución del tratamiento informativo que los cibermedios hacen de un concepto concreto, desigualdad económica en este caso, en el nuevo ecosistema híbrido definido por Chadwick (2013).

Los resultados obtenidos por la propia investigación en el desarrollo de herramientas metodológicas previas han permitido determinar la necesidad de extender el periodo de análisis con el objetivo de poder establecer el recorrido del término en la agenda cibermediática. Esta nueva aproximación facilita profundizar en el comportamiento de esta problemática en los medios de comunicación, de tal manera que no se ofrezca, simplemente, la fotografía de un momento específico, propia de las investigaciones transversales; sino que se pro- 
fundice en el ciclo de atención mediática y en el proceso de visibilización de la desigualdad económica, mediante un estudio de carácter longitudinal.

Además, el adaptar el estudio para que se adecue a las características de otros contextos nacionales permite establecer una comparación entre el comportamiento de los medios de comunicación españoles al abordar esta problemática frente al desarrollo de la misma en las agendas mediáticas de otros países.

De este modo, el objetivo principal se alcanza, diseñando un instrumento que estudiará el término en su totalidad, por dos motivos principales. Se trata de una herramienta de carácter longitudinal (2009-2018), lo cual permite medir los cambios asociados al desarrollo de la profesión periodística en el nuevo espacio mediático que supone el sistema híbrido de medios. Por otro lado, al seleccionar una muestra de medios de cuatro países se obtendrán resultados no sólo válidos para el contexto español, sino para el ámbito internacional. Este carácter supranacional resulta altamente pertinente en relación con el estudio de un concepto que, desde su nacimiento, y en su posterior vinculación a la crisis económica iniciada en 2008, siempre ha sido global.

No obstante, existen algunos aspectos susceptibles de mejora, ya que del análisis de contenido pueden derivar otros más específicos, tales como: análisis del discurso o análisis de la relación de la desigualdad económica con protestas sociales como Occupy Wall Street, 15M, Primavera Árabe o Chalecos Amarillos.

Además, en una segunda fase, los resultados obtenidos se complementarán con la realización de entrevistas en profundidad para obtener una visión completa de los procesos, paliando las plausibles deficiencias del análisis de contenido, centrada en el producto final. No obstante, es importante recalcar la necesidad de la triangulación de investigación, asumiendo la definición de Denzin (1970), para obtener conclusiones sólidas respecto al cambio radical vivido en los medios; comenzando, en este caso, por el análisis de contenido.

Por último, cabe destacar que durante la configuración de esta propuesta se encontró cierto obstáculo que si bien ha sido solventados se estima pertinente explicitar. Este inconveniente se encuentra entroncado directamente con los cibermedios. En cuanto a la selección final de medios para el análisis; en un principio se consideró contar con nativos digitales, en contraposición a los legacy media generalistas y los especializados, para poder cubrir todo el espectro mediático en relación con su naturaleza. Sin embargo, esta idea fue desechada, puesto que, al haber configurado un espacio temporal de 10 años para poder observar la evolución, se descubrió que varios de los nativos habían surgido con fecha posterior. Asimismo, a lo largo de la fase del trabajo de campo es posible que haya que reconfigurar el tipo de muestreo, en función de la relevancia del concepto en las publicaciones de cada uno de los cibermedios.

\section{Notas al final}

1. En el caso de los medios de habla inglesa se opta por "income inequality"; mientras que en los medios de habla francesa se opta por el término "inégalités des revenus".

2. En 10 de los productos periodísticos analizados no aplicaba el ámbito geográfico

3. El periodista autor del texto menciona el concepto de desigualdad económica en 35 casos (26,1\%); habiendo un total de 134 menciones al término en las 128 noticias analizadas.

4. Hay un alto número de informaciones donde el tema principal no ha sido categorizado de manera específica y aparece en la opción "Otros", 24,2 \%. En estos casos se vincula a cuestiones políticas, pero también a otros temas no relacionados tradicionalmente con la desigualdad económica: arte, ciencia y tecnología, deportes...

\section{Referencias}

Alencar, A., y Kruikemeier, S. (2018). Audiovisual infotainment in European news: A comparative content analysis of Dutch, Spanish, and Irish television news programs. Journalism, 19(11), 1534-1551. https:// doi.org/10.1177/1464884916671332

Anderson, C. W., Bell, E., y Shirky, C. (2012). Post-Industrial Journalism: Adapting to the Present [web]. https://academiccommons.columbia. edu/doi/10.7916/D8N01JS7

Aruguete, N. (2015). El poder de la agenda: Política, medios y público. Biblos.

Asociación de la Prensa de Madrid (2018). Informe Anual de la Profesión Periodística [web]. https://www.apmadrid.es/wp-content/ uploads/2019/07/Informe-profesi\%C3\%B3n-2018_baja.pdf

Becker, B. y Wehner, J. (2001). Electronic Networks and Civil Society. En Ess, C. (Ed.). Culture, Technology, Communication: Towards an Intercultural Global Village (pp. 67-86). SUNY Press.

Benkler, Y. (2006). The wealth of networks: How social production transforms markets and freedom. Yale UP.

Berrocal Gonzalo, S., Redondo García, M. y Campos Domínguez, E. (2014). La presencia del infoentretenimiento en los canales generalistas de la TDT española. Revista Latina de Comunicación Social, (69), 85-103.

Bhargava, R. (2009). Manifesto For The Content Curator: The Next Big SocialMedia Job Of The Future? [web]. https://www.rohitbhargava. com/2009/09/manifesto-for-the-content-curator-the-next-big-social-media-job-of-the-future.html

Bruns, A. y Highfield, T. (2015). From news blogs to news on Twitter: Gatewatching and collaborative news curation. En: Coleman, S. y Freelon, D. Handbook of digital politics (pp. 325-339). Edward Elgar Publishing.

Chadwick, A. (2013). The Hybrid Media System. Politics and Power. Oxford University Press.

Dearing, J. W., y Rogers, E. M. (1996). Agenda-Setting. Sage. 
Denzin, N.K. (1970). Sociological Methods: a Source Book. Aldine Publishing Company.

Diel, S. (2017). New Media, Legacy Media and Misperceptions Regarding Sourcing. KOME - An International Journal of Pure Communication Inquiry, 5(1), 104-120. https://doi.org/10.17646/KOME.2017.17

Downs, A. (1972). Up and down with ecology-The issue-attention cycle. Public interest, 28, 38-50.

Entman, R. M. (1993). Framing: Toward Clarification of a Fractured Paradigm. Journal of Communication, 43(4), 51-58. https://doi. org/10.1111/j.1460-2466.1993.tb01304.x

Galtung, J., y Ruge, M. H. (1965). The structure of foreign news: The presentation of the Congo, Cuba and Cyprus crises in four Norwegian newspapers. Journal of peace research, 2(1), 64-90.

Gamson, W. A., y Modigliani, A. (1987). The changing culture of affirmative action. En: Braungart, R. (Ed.). Research in political sociology (pp. 137-177). Jai Press.

García-Clemente, J.V., Clemente-Fernández, M.D., y López-Aboal, M. (2013). La organización de las redacciones en los nuevos diarios digitales españoles y su relación con los nuevos modelos de negocios. Textual y Visual Media, 6, 141-160. http://www.textualvisualmedia. com/index.php/txtvmedia/article/view/81/66

Golding, P., y Elliott, P. (1979). Making the news (Vol. 8). Longman.

Gross, K. (2008). Framing persuasive appeals: Episodic and thematic framing, emotional response, and policy opinión. Political Psychology, 29(2), 169-192. https://doi.org/10.1111/j.1467-9221.2008.00622.x

Guallar, J; Leiva-Aguilera, J. (2013). El content curator. Guía básica para el nuevo profesional de internet. UOC.

Harcup, T., y O'Neill, D. (2017). What is News? Journalism Studies, 18(12), 1470-1488. https://doi.org/10.1080/1461670X.2016.1150193

Hester, J. B., y Dougall, E. (2007). The efficiency of constructed week sampling for content analysis of online news. Journalism and Mass Communication Quarterly, 84(4), 811-824. https://doi. org/10.1177/107769900708400410

Kiousis, S. (2004). Explicating media salience: A factor analysis of New York Times issue coverage during the 2000 U.S presidential election. Journal of Communication, 54(1), 71-87. https://doi.org/10.1093/ joc/54.1.71

Krippendorff, K. (2004). Content Analysis. An Introduction to Its Methodology. Sage.

Kuznets, S. (1955). Economic Growth and Income Inequality. The American Economic Review, 45(1), 1-28.

Labio-Bernal, A. (2008). Periodismo de entretenimiento: La trivialización de la prensa de referencia. Estudios sobre el mensaje periodístico, 14, 435-447.

Logan, R. (2010). Understanding New Media: Extending Marshall McLuhan. Peter Lang.

Lozano, J.C. (2004). Infotainment in national TV news: A comparative analysis of Mexican, Canadian and U.S. news programs. Actas de la Annual Conference of the International Association for Media and Communication Research. IAMCR/AIERI.

Masip, P.; Ruiz-Caballero, C.; Suau, J. (2019). Active audiences and social discussion on the digital public sphere. Review article. El profesional de la información, 28(2), https://doi.org//10.3145/epi.2019. mar.04
McKay, A. (2002). Defining and measuring inequality (No 1) [web] https://www.odi.org/sites/odi.org.uk/files/odi-assets/publications-opinion-files/3804.pdf

McCall, L. (2015). American Beliefs about Income Inequality: What, When, Who and Why? En A. S. Wharton (Ed.). Working in America: Continuity, Conflict, and Change in a New Economic Era (pp.185-200). Routledge.

McCombs, M. (2006). Estableciendo la agenda: El impacto de los medios en la opinión pública y en el conocimiento. Paidós.

McCombs, M., y Evatt, D. (1995). Los temas y los aspectos: Explorando una nueva dimensión de la agenda setting. Comunicación y sociedad, 8(1), 7-32.

Milanović, B. (2010). The Haves and the Have-Nots: A Brief and Idiosyncratic History of Global Inequality. Basic Books.

Milanović, B. (2016). Global inequality: A new approach for the age of globalization. Harvard University Press.

Mompart, J. L. (2009). From quality journalism to speculative journalism. Transfer: Journal of Contemporary Culture, 4, 51-61.

Neuendorf, K. A. (2002). The Content Analysis Guidebook. Sage.

O'Reilly, T. (2009). What is Web 2.0. O'Reilly Media.

Odriozola-Chéné, J., Pérez-Arozamena, R., y Fernández-Planells, A. (2019). El papel de los legacy media en la construcción de la opinión pública: conceptualización, enfoques y participación de las audiencias en el tratamiento de la desigualdad económica en seis cibermedios españoles. XXV Congreso Internacional de la Sociedad Española de Periodística: Oportunidades y riesgos del periodismo hiperconectado, Bilbao, España.

Otto, L., Glogger, I., y Boukes, M. (2017). The Softening of Journalistic Political Communication: A Comprehensive Framework Model of Sensationalism, Soft News, Infotainment, and Tabloidization. Communication Theory, 27(2), 136-155. https://doi.org/10.1111/ comt.12102

Pérez-Altable, L., Pérez-Arozamena, R., Linares-Lanzman, J., Odriozola-Chéné, J., Fernández-Planells, A., Serrano-Tellería, A., y Díaz-Noci, J. (2020). The Crisis, the People and the Media: How Digital Public Opinion Debates on Economic Inequality. En Peña-Fernández, S., Meso-Ayerdi, K. y Larrondo Ureta, A. (Eds.), Active Audiences. Empowering Citizens' Discourse in the Hybrid Media System (pp.131-143). McGraw-Hill.

Petterson, M. A. (2003). Anthropology y Mass Communication: Media and Myth in the New Millennium. Berghahn Books

Philips, A. (2010). Old Sources: New Bottles. En N. Fenton (Ed.), New media, Old News: Journalism and Democracy in the Digital Age (pp. 87-101). Sage.

Piketty, T. (2013). Le Capital au XXIe siècle. Seuil.

Ray, D. (1998). Development Economics. Princeton University Press.

Redden, J. (2011). Poverty In The News. Information, Communication y Society, 14(6), 820-849. https://doi.org/10.1080/1369 $118 \times .2011 .586432$

Seligson, M. A., y Passé-Smith, J. T. (2008). Development and Underdevelopment: The Political Economy of Global Inequality. Lynne Rienner.

Semetko, H. A., y Valkenburg, P. M. (2000). Framing European politics: A content analysis of press and television news. Journal of communication, 50(2), 93-109. https://doi.org/10.1111/j.1460-2466.2000. 
tb02843.x

Sjøvaag, H., y Stavelin, E. (2012). Web media and the quantitative content analysis: Methodological challenges in measuring online news content. Convergence: The International Journal of Research into New Media Technologies, 18(2), 215-229. https://doi. org/10.1177/1354856511429641

Soroka, S. N. (2002). Issue attributes and agenda-setting by media, the public, and policymakers in Canada. International Journal of Public Opinion Research, 14(3), 264-285. https://doi.org/10.1093/ ijpor/14.3.264

Stiglitz, J. (2012). The Price of Inequality. Penguin.

Strodthoff, G. G., Hawkins, R. P., y Schoenfeld, A. C. (1985). Media Roles in a Social Movement: A Model of Ideology Diffusion. Journal of Communication, 35(2), 134-153. https://doi. org/10.1111/j.1460-2466.1985.tb02239.x

The Nobel Prize. (n.d.a). Award ceremony speech [web]. https://www. nobelprize.org/prizes/economic-sciences/1971/ceremony-speech/

The Nobel Prize. (n.d.b). The Sveriges Riksbank Prize in Economic Sciences in Memory of Alfred Nobel 1971 [web]. https://www.nobelprize.org/prizes/economic-sciences/1971/summary/

Thompson, J. B., (2005). The New Visibility. Theory, Culture y Society, 22(6), 31-51. https://doi.org/10.1177/0263276405059413

Thompson, J. B., (1998). Los Media y la modernidad: una teoría de los medios de comunicación. Paidós.

Thrall, A. T., Lollio-Fakhreddine, J., Berent, J., Donnelly, L., Herrin, W., Paquette, Z., ... Wyatt, A. (2008). Star Power: Celebrity Advocacy and the Evolution of the Public Sphere. The International Journal of Press/ Politics, 13(4), 362-385. https://doi.org/10.1177/1940161208319098

Toffler, A. (1980). The third wave. William Morrow.

Tuchman, G. (1978). Making News: A Study in the Construction of Reality. The Free Press.

Westlund, O. (2013). Mobile news: A review and model of journalism in an age of mobile media. Digital Journalism, 1(1), 6-26. https://dx.doi. org/10.1080/21670811.2012.740273

Wilkinson, R. G., y Pickett, K. (2009). The Spirit Level. Why More Equal Societies Almost Always Do Better. Allen Lane.

Zhu, J. H. (1992). Issue competition and attention distraction: A zero-sum theory of agenda-setting. Journalism Quarterly, 69(4), 825-836. https://doi.org/10.1177/107769909206900403

\section{CV}

Rosa Pérez Arozamena. Doctora en Ciencias de la Comunicación por la Universidad Antonio de Nebrija (2016), Licenciada en Periodismo (Universidad de Sevilla, 2008), tiene un máster en radio y televisión ( $U$. Nebrija, 2010). Profesora contratada doctora en la Universidad Europea del Atlántico (Santander, España), compatibiliza además la docencia en las universidades Nebrija, Camilo José Cela y UCAM. Su línea de investigación aborda las transformaciones del mundo periodístico: nuevas rutinas, fenómenos de infoentretenimiento y espectacularización informativa, entre otros. https://www.linkedin.com/in/dra-rosa-pérez-arozamena-49101044/

Javier Odriozola Chéné. Doctor en Periodismo por la Universidad del País Vasco (España). Profesor contratado doctor a tiempo completo de la Universidad Europea del Atlántico (Santander, España) y director académico del grado en Periodismo. Investigador en proyectos sobre configuración de las agendas mediáticas, influencias en el desarrollo de la profesión periodística, periodismo digital, periodismo ambiental y consumo de Internet. Miembro de comités científicos de revistas indexadas, revisor de artículos de investigación y editor de Comhumanitas (2014 - 2017). https://www.researchgate.net/ profile/Javier_Odriozola-Chene

\section{ocm \\ Observatorio de Cibermedios}

\section{https://observatoriocibermedios.upf.edu/}
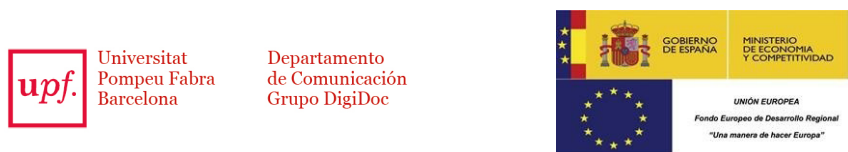

El Observatorio de Cibermedios es una producción del Grupo de Investigación en Documentación Digital y Comunicación Interactiva (DigiDoc) del Departamento de Comunicación de la Universitat Pompeu Fabra.

El Observatorio de Cibermedios (OCM) forma parte del proyecto del Plan Nacional "Creación y contenido interactivo en la comunicación de información audiovisual: audiencias, diseño, sistemas y formatos". CSO2015-64955-C4-2-R (MINECO/ FEDER), Ministerio de Economía y Competitividad (España). 